\title{
On the Number of Anchor Nodes for the Localization of Smart Energy Meters (SEM) in an African Environment
}

\author{
Griffith S. Klogo \\ Department of Computer \\ Engineering, KNUST \\ Kumasi, Ghana
}

\author{
James D. Gadze \\ Department of Electrical and \\ Electronics Engineering, \\ KNUST, Kumasi, Ghana
}

\author{
Henry Nunoo-Mensah \\ Department of Computer \\ Engineering, KNUST \\ Kumasi, Ghana
}

\begin{abstract}
The number of anchor nodes required for accurate localization is an important problem in the wireless sensor network research community. The error associated with localization is high when anchor nodes are not optimally placed in the network. No matter how the network is set up, the error associated with localization is inevitable. There are various sources for these errors, one of which is the unavailability of anchor nodes. These conditions arise due to stumpy deployment density or poor signal propagation owing to factors like multipath effects, fading effects and poor visibility. This paper proposes a method of determining the minimum number of anchor nodes required for a given sensor (smart energy meter) network dimension using triangulation as the localization process. The proposed method uses the sensitivity of the sensor nodes and various environmental conditions. Using the sensitivity of the sensor nodes and the environmental conditions, the minimum number of anchor nodes for a network dimension was determined through simulation. The minimum number of anchors required for a network with clear line of sight, sub-urban, residential and non-line of sight environments was achieved with this method.
\end{abstract}

\section{Keywords}

Anchor node, shadowing, sensitivity, smart energy meter (SEM), outage probability.

\section{INTRODUCTION}

Wireless sensor networks consist of distributed autonomous sensors that monitor physical properties in an environment. The advancement in modern technologies in manufacturing sensor nodes has led to their large scale deployment for numerous applications. Smart metering is one application area that has benefited from the advancements in wireless sensor network technologies. Smart energy meters employ special measuring sensor, wireless communication and computation module suitable for modern energy management. Smart energy meters in modern energy management helps in reducing distribution cost and load management. These smart energy meters are deployed in households, industries and other facilities to measure energy consumption of these infrastructures for billing and energy management purposes. In Sub-Sahara environments, the poor planning of settlements [1] makes locating and monitoring of deployed smart meters a challenge. The utility provider usually is unable to visit the facilities of consumers regularly. Fraud, illegal connections and tampering of energy meters are difficult to detect in good time. In smart metering, knowing the location of the meters helps in reducing the commercial loss and the cost of sending personnel to read power consumption of customers. Location information of smart energy meters also helps in energy budget planning and monitoring the power usage of facilities.
This paper uses wireless sensor network anchor-based localization technique in locating smart energy meters. The process of localization is finding the positional coordinates of smart energy meters manually or automatically with reference to anchor nodes [2] [3] [4]. Anchor-based localization is estimating the location of any smart energy meter with reference to other smart energy meter (called anchor nodes) whose location is known. Anchor nodes are nodes that are aware of their positional coordinates in the network, that is, their position in the network is known by means of GPS or the position has been hard-coded into the node [5] [6]. The location and number of anchor nodes does influence the accuracy of the localization process [2]. Anchor nodes play important roles in determining absolute or relative coordinates of the smart energy meters in WSN localization technique. Research has established that the presence of anchors in the network for localization does increases the degree of accuracy in estimating the positional coordinates on the unknown node(s) and that increasing the number of anchor nodes may minimize the localization error, but with a draw back on cost of setting up such a network [2] [3]. Anchor-based localization techniques have a high degree of accuracy compared to anchor free localization techniques [2] [6] [7]. Anchor-based localization of smart energy meters is by virtue of the proximity of the smart energy meters to the anchor node(s) or by estimating the distance of the smart energy meters from the anchor node(s) [6] [7] [8]. The anchor-based localization technique used in this paper employs ranging to estimate the location of the smart energy meters. Range-based localization involves measuring the distance between two nodes to determine the location of an unknown node in the network. Range-based localization has more accuracy compared to range-free techniques [2] [8]. Range-based techniques for localization use wireless connectivity and topology to evaluate the location of unknown sensor nodes (smart energy meters) [8] [9]. Wireless connectivity and topology are influenced by environmental conditions. Effective range based localization depends on the smart energy meters having enough signal strength from anchor nodes to estimate their location [8]. Sensor nodes having good signal strength will mean the network having enough radio coverage across the network. Smart energy meter network with good radio coverage from anchor nodes has high localization accuracy. Radio coverage has different characteristics in line of sight and non-line of sight environments. Signal strengths are affected by hardware and environmental factors such as urban, sub-urban, and rural settlements. Smart energy meters are deployed in different environments to obtain energy data to estimate the amount of energy consumed by customers for billing purposes and energy budgets for utility service providers. For high accurate localization of smart energy meters, factors such as optimal anchor placements and network structure is required. 
Localization of smart energy meters in an urban, sub-urban and rural environment requires a good knowledge of different anchor node density and placements strategies for accuracy. In this paper, the radio coverage of anchor nodes in a square perimeter is simulated to determine the minimum number of anchor nodes required for different environments. Based on the method used, the minimum number of anchor nodes for different network sizes in different environments is achieved.

\section{ANCHOR NODE DENSITY AND PLACEMENT}

No matter how the network is set up, the error associated with localization cannot be avoided. There may be various sources for this error, one of which is the unavailability of anchor nodes. This condition may arise due to stumpy deployment density or poor signal propagation owing to factors like multipath effects, fading effects and poor visibility. Radio signals behave differently in different environments, the distance of a radio signal being transmitted depends on transmitter power, antenna gain for both the transmitter and receiver and the receiver's radio sensitivity. Receiver sensitivity is the minimum signal power level (in $\mathrm{dBm}$ or $\mathrm{mW}$ ) that is necessary for the receiver to accurately decode a given signal [10] [11]. Sensitivity is purely a receiver specification and is independent of the transmitter. The placement and spacing of the anchor nodes will be influenced by the receiver sensitivity of the smart energy meters and the environment of the network (urban, sub-urban or rural). The number of anchor nodes deployed in a network will also be dependent on the network environment, size and sensitivity of the nodes. Table 1 , shows a typical specification of a sensor node (specifically MICAz) [12].

Table 1: Typical Specification of a sensor node [12]

\begin{tabular}{|l|l|}
\hline \multicolumn{2}{|c|}{ RF Transceiver } \\
\hline Frequency band & $2400 \mathrm{Mhz}$ to $2483.5 \mathrm{MHz}$ \\
\hline Transmit (TX) data rate & $250 \mathrm{kbps}$ \\
\hline RF power & $-24 \mathrm{dBm}$ to $0 \mathrm{dBm}$ \\
\hline Receiver Sensitivity & $-90 \mathrm{dBm}$ (min.), -94dBm (Typ.) \\
\hline Outdoor Range & $75 \mathrm{~m}$ to $100 \mathrm{~m}$ \\
\hline Indoor Range & $20 \mathrm{~m}$ to $30 \mathrm{~m}$ \\
\hline
\end{tabular}

\section{SYSTEM MODEL}

The number of anchor nodes for network coverage depends on size of the network (perimeter), sensitivity of receivers for both anchor and sensor nodes (smart energy meters) and the environmental conditions in the network. A signal transmitted via a wireless channel will naturally experience random deviation due to obstruction from objects in the signal path, giving rise to random variations of the received power at a given distance (d) [14]. It is important that a model that factors these effects is considered for this research. This research used the combined path loss and shadowing model as shown in equation 1 to estimate the minimum number of anchor nodes required for a given network size. The path loss obeys the simplified path-loss model with $\mathrm{K}=-35.54 \mathrm{~dB}$ and the path-loss exponent $\gamma$ and the shadowing obeys the Gaussian log-normal model $(\psi \mathrm{dB})$ with mean given by the path-loss model and standard deviation $\sigma_{\psi d B}$ [14].
$\frac{P_{r}}{P_{t}} d B=10 \log _{10} K-10 \gamma \log _{10} \frac{d}{d_{O}}-\psi_{d B}$

where,

$\psi_{d B}=10 \log _{10} \psi$ and $\psi=P_{t} / P_{r}$

For simulation the following conditions were considered to estimate the number and spacing of anchor nodes.

- Perimeter placement of anchor nodes in a 100by100meter square area

- $\quad$ Frequency band of 2.4GHz IEEE 802.15.4 standard

- Log-Normal Shadowing with Gaussian distribution

- If the transmitted power $(\mathrm{Pt})$ is a Gaussian random variable then the received power $(\mathrm{Pr})$ is such that $\mathrm{Pr}$ $=\log \mathrm{Pt}$, then the transmitted power $(\mathrm{Pr})$ is a $\log$ normal random variable [13]. Shadowing is $\log$ normal shadowing when the path loss in $\mathrm{dB}$ is Gaussian as expressed in equation 1 [14] [15].

- $\quad$ Friis equation of path loss [16] [17]

With the above conditions, the distance travelled by anchor signals were simulated to estimate the number and placement of anchor nodes that will be needed for various environmental conditions. Using the combined path loss and shadowing model as shown in equation 1 , the environmental conditions for line-of-sight (LOS), Sub-urban, Residential and non-lineof-sight (nLOS) were simulated to estimate appropriate spacing of anchor nodes. Table 2 show the parameters used in the simulation.

\subsection{Outage Probability of the Anchor Nodes}

The combined effect of the path loss and shadowing has important implications on the smart meter network [14]. Therefore in determining the minimum number of anchor nodes needed for localization, it is important to know the minimum received power level $P_{\min }$ below which performance of anchor nodes becomes unacceptable. $P_{\min }$ is the power level at which the smart meters are unable to determine their location with reference to the anchor nodes. Outage probability [14] $P_{\text {out }}\left(P_{\text {min }}, d\right)$ under path loss and shadowing is the probability that the received power at a given distance $\mathrm{d}$, $P_{r}(d)$, fall below $P_{\text {min }}: P_{\text {out }}\left(P_{\text {min }}, d\right)=p\left(P_{r}(d)<P_{\text {min }}\right)$ as shown in equation 2 [14]. An outage probability of $1 \%$ is most desirable in wireless systems. This research used the outage probability to estimate the failure of anchor nodes in the localization process.

$p\left(P_{r}(d) \leq P_{\min }\right)=1-Q\left(\frac{P_{\min }-\left(P_{t}+10 \log _{10} K-10 \gamma \log _{10}\left(d / d_{O}\right)\right)}{\sigma_{\psi_{d B}}}\right)$

Table 2: Simulation Parameters

\begin{tabular}{|l|l|}
\hline Frequency band & 2400Mhz to $2483.5 \mathrm{MHz}$ \\
\hline RF power & $-24 \mathrm{dBm}$ to $0 \mathrm{dBm}$ \\
\hline Receiver Sensitivity & $-90 \mathrm{dBm}$ (min.), -94dBm (Typ.) \\
\hline Transmitter Gain (dB) & $1 \mathrm{~dB}$ \\
\hline Receiver Gain (dB) & $1 \mathrm{~dB}$ \\
\hline Max. Distance & $100 \mathrm{~m}$ \\
\hline
\end{tabular}




\begin{tabular}{|l|ll|}
\hline Reference Dist. of Rx & $1 \mathrm{~m}$ & \\
\hline Path Loss Exponent (n) [5] & 2 & (line of sight) \\
& 2.8 & (Sub-urban area) \\
& 2.93 & (Residential area) \\
& 3.5 & (Non-line of sight) \\
\hline $\begin{array}{l}\text { Gaussian random variable } \\
\text { with standard deviation }(\sigma)\end{array}$ & $9 \mathrm{~dB}$ & (line of sight) \\
[5] & $7.85 \mathrm{~dB}$ & (Sub-urban area) \\
& $11.7 \mathrm{~dB}$ & (Residential area) \\
& 0 & \\
\hline $\begin{array}{l}\text { Gaussian random variable } \\
\text { mean }\end{array}$ & 0 & \\
\hline
\end{tabular}

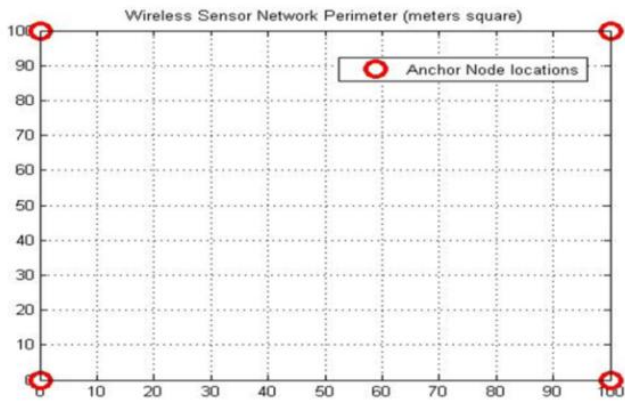

Fig 1: Square perimeter of $100 \times 100$ meters

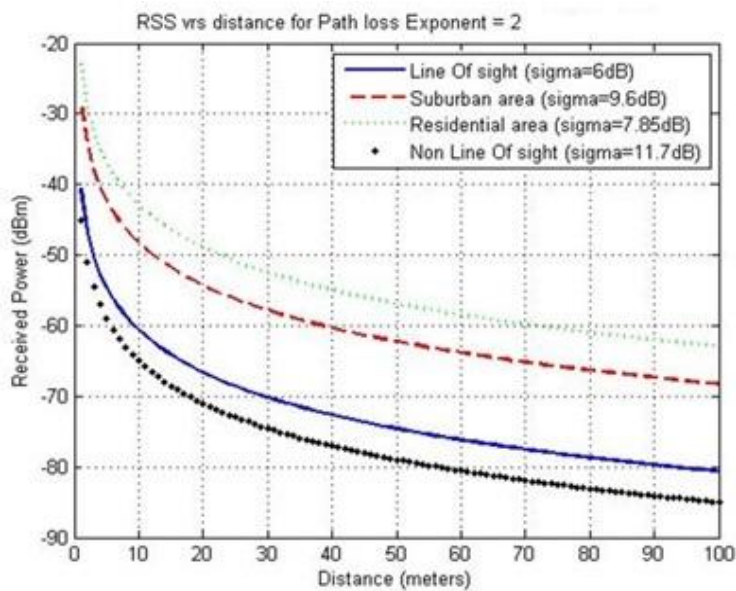

Fig 2: RSS verses distance for path loss exponent $=2$

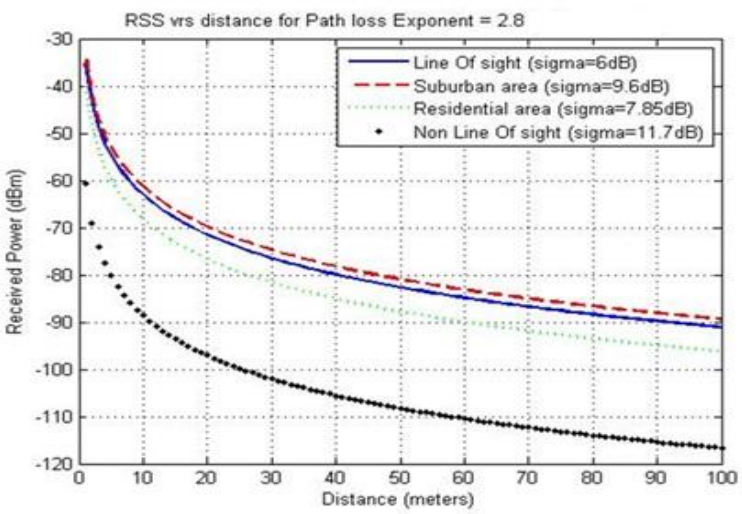

Fig 3: RSS verses distance for path loss exponent $=\mathbf{2 . 8}$

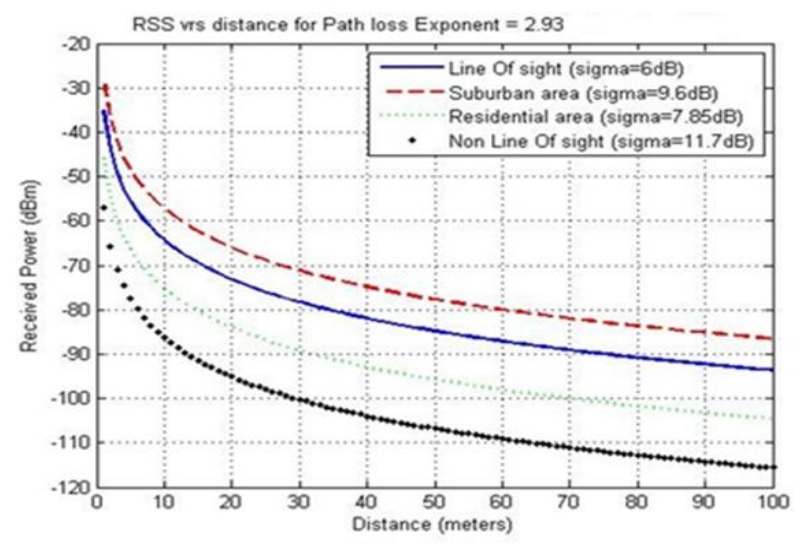

Fig 4: RSS verses distance for path loss exponent $=\mathbf{2 . 9 3}$

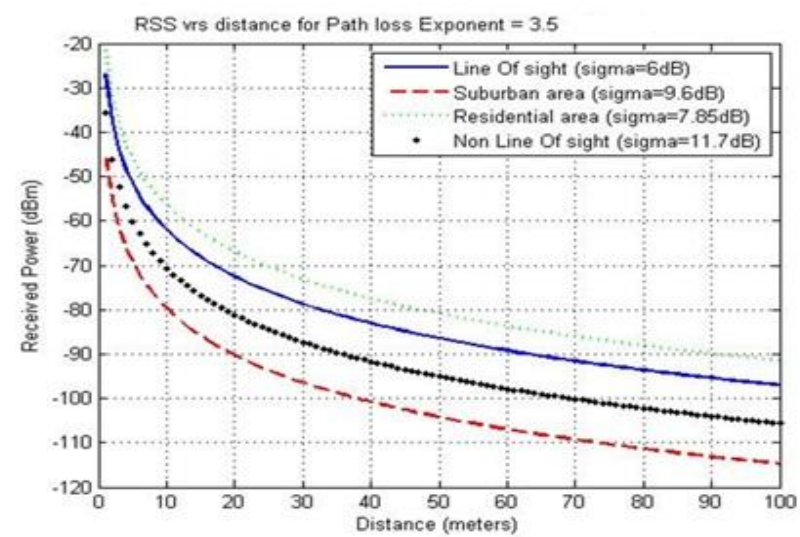

Fig 5: RSS verses dist. for path loss exponent $=3.5$

\section{DISCUSSION OF RESULTS}

Radio signal strength (RSS) drops with distance travelled from transmitter to receiver. For the simulation, this paper considered the transmit power range of $-24 \mathrm{~dB}$ to $0 \mathrm{~dB}$. The signal strength was observed while reducing the distance travelled by the signal in the environmental conditions for line of sight, sub-urban, residential and non-line of sight. In a 100x100 meters square perimeter, signals from one anchor travelling diagonally to another anchor in the opposite diagonal will have to travel about 150 meters. In figure 2 , the path loss exponent for line of sight and typical Gaussian random variable for the shadowing effects for line of sight, sub-urban, residential and non-line of sight with standard deviation (sigma) values of $6 \mathrm{~dB}, 9.6 \mathrm{~dB}, 7.85 \mathrm{~dB}$ and $11.7 \mathrm{~dB}$ respectively was used. Comparing the results in figure 2 to typical specifications of a sensor nodes in table 1, it implies that a minimum of 3 anchor nodes will be needed to estimate the location of any number of unknown nodes (SEM) within the perimeter using triangulation. The signal strength of about $-85 \mathrm{~dB}$ at 100 meters away from the transmitter is more than the typical sensitivity in table 1 . Path loss exponent for a suburban area in figure 3 shows some drop in the distance the radio signal can travel for it to be detected by receiving nodes (SEM). In line of sight, sub-urban and residential environments the signals can travel to about 70meters to be detected by the receiving nodes with sensitivity of $-94 \mathrm{~dB}$ but the same signal can only be detected just 15 meters from the transmitter in non-line of sight environments. In figure 4, path loss exponent for residential areas was used and the result shows typical performance as in sub-urban environment with detection range increasing to about 20meters in non-line of sight. Figure 5 shows results for the simulation with path loss 
exponent for non-line of sight. The signal can be detected in a range of 20meters for a sub-urban area, 50 meters for non-line of sight area and about 100meters for both residential and line of sight areas. Clearly, with the minimum number of anchor nodes in the network it is possible to locate all smart energy meters within the perimeter as shown in table 3 . To verify the reliability and availability of the anchor nodes in the localization process, an outage probability was performed. Outage probabilities for line of sight, sub-urban residential and Non-line of sight environment are $4.64 \mathrm{e}-5 \%, 1.33 \%$, $0.80 \%$ and $12.44 \%$ respectively. This is an indication that the anchor nodes in their respective perimeter size are reliable since their outage probability is less or just a little above $1 \%$ excluding that of non-line of sight. Localization can still be done by increasing the number of anchor nodes to five (5) even though the outage probability is high. With the high number of anchor nodes the outage probability will be reduced.

\section{CONCLUSION}

High accuracy of range based localization techniques depends largely on the number and placement of anchor nodes in the network. In this paper, the placement and minimum number of anchor nodes required for localization of smart energy meters was determined based on the sensitivity of receiving nodes in the network considering various environmental factors such as an area with clear line of sight, sub-urban area, residential area and an area where sensor nodes do not have clear line of sight. The results from the simulations show that anchor nodes can be optimally placed for accurate localization. Based on these results we intend to develop a sophisticated communications infrastructure for locating and monitoring smart energy meters deployed in typical environments in sub-Saharan Africa. Coverage analysis will be performed to verify the reliability of the positions of the anchor nodes in the localization process. Table 3 summarizes the results for the minimum number of nodes and the spacing for a square perimeter placement for the typical sensor node specification in table 1 .

Table 3: Summary of results

\begin{tabular}{|l|l|l|l|}
\hline Environment & $\begin{array}{l}\text { Perimeter } \\
\text { Size }\end{array}$ & $\begin{array}{l}\text { Min. No. } \\
\text { of Anchor } \\
\text { Nodes }\end{array}$ & $\begin{array}{l}\text { Outage } \\
\text { Probability for } \\
\text { each node(\%) }\end{array}$ \\
\hline Line of sight & $150 \times 150 \mathrm{~m}$ & 3 nodes & $4.64 \mathrm{e}-05 @ 150 \mathrm{~m}$ \\
\hline Sub-urban & $70 \times 70 \mathrm{~m}$ & 4 nodes & $1.33 @ 70 \mathrm{~m}$ \\
\hline Residential & $70 \times 70 \mathrm{~m}$ & 4 nodes & $0.80 @ 70 \mathrm{~m}$ \\
\hline $\begin{array}{l}\text { Non-line of } \\
\text { sight }\end{array}$ & $50 \times 50 \mathrm{~m}$ & 5 nodes & $12.44 @ 50 \mathrm{~m}$ \\
\hline
\end{tabular}

\section{REFERENCES}

[1] Collins Adjei Mensah, Mphil Thesis on "Causes and Consequences of Informal Settlement Planning in Ghana: A Case Study of Aboabo, A Suburb of Kumasi Metropolis", Department of Geography and Regional Planning of the Faculty of Social Sciences, University of Cape Coast.

[2] G. S. Klogo and J. D. Gadze, "Energy Constraints of Localization Techniques in Wireless Sensor Networks (WSN): A Survey" International Journal of Computer Applications (0975 - 8887), Vol. 75- No.9, Aug. 2013, pp 44-52Fröhlich, B. and Plate, J. 2000. The cubic mouse: a new device for three-dimensional input. In
Proceedings of the SIGCHI Conference on Human Factors in Computing Systems.

[3] U. Nazir, M. A. Arshad, N. Shahid and S.H. Raza, "Classification of Localization Algorithms for Wireless Sensor Network: A Survey", 2012 International Conference on Open Source Systems and Technologies (ICOSST), pp 1-5

[4] Z. Zhong and T. He, Member, IEEE, "RSD: A Metric for Achieving Range-Free Localization beyond Connectivity", IEEE Transaction on Parallel and Distributed Systems, Vol. 22, NO. 11, Novermber 2011, pp 1943-1951.

[5] S. Tian, X. Zhang, X. Wang, P. Sun and H. Zhang, "A Selective Anchor Node Localization Algorithm for Wireless Sensor Networks", 2007 International Conference on Convergence Information Technology, pp 358-362.

[6] Chia-Ho Ou, Member, IEEE and Wei-Lun He, "Path Planning Algorithm for Mobile Anchor-Based Localization in Wireless Sensor Networks", IEEE Sensor Journal, Vol. 13, NO. 2, February 2013, pp 466-475

[7] N. Salman, H. K. Maheshwari, A.H. Kemp and M. Ghogho, "Effects of anchor placement on mean-CRB for localization", 2011 The 10th IFPI Annual Mediterranean Ad Hoc Networking Workshop, pp 115-118

[8] Y. Wang, X. Wang, D. Wang, Members, IEEE and D. P. Agrawal, Fellow, IEEE, "Range-Free Localization Using Expected Hop Progress in Wireless Sensor Networks", IEEE Transaction on Parallel and Distributed Systems, Vol. 20, NO. 10, October 2009, pp 1540-1552

[9] B. Yao, W. Wang and Q. Yin, "Angle of Departure Aided Sensor Localization Technique under Multipath Environment", Globecom 2012, Ad-Hoc and Sensor Network Symposium, pp 304-308

[10] Matt Loy, "Understanding and Enhancing Sensitivity in Receivers for Wireless Applications", Texas Instruments Technical Brief SWRA030.

[11] Receiver Sensitivity, http://www.digi.com/technology/rfarticles/receiver-sensitivity, accessed on 30th September 2013.

[12] Micaz datasheet, Crossbow Technology, Inc., Document Part Number: 6020-0060-04 Rev A

[13] T.S. Rappaport, Wireless Communications, Prentice Hall, 1996

[14] Andrea Goldsmith, Wireless Communications, Cambridge university press, 2005

[15] Simon R. Saunders, Antennas and Propagation for Wireless Communication Systems, John Wiley and Sons, LTD, 1999.

[16] Fading and shadowing, http://morse.colorado.edu/ tlen5510/text/classwebch4.ht $\mathrm{ml}$, accessed on 18th September 2013.

[17] Shadowing, JPL's Wireless Communication Reference Website, http://www.wirelesscommunication.nl/reference/chaptr0 3/shadow/shadow.htm, accessed on 30th September 2013. 